\title{
Ground Control Point Generation from Simulated SAR Image Derived from Digital Terrain Model and its Application to Texture Feature Extraction
}

\author{
Kohei Arai \\ Faculty of Science and Engineering \\ Saga University, Saga City \\ Japan
}

\begin{abstract}
Ground Control Point: GCP generation from simulated topographic map derived from Digital Terrain Model: DTM is proposed. Also, texture feature extraction is attempted from the simulated image. In this study, simulated image is derived from elevation data only, under assumptions of a simple scattering model without consideration of complex dielectric constant of the targets of interest. The performance of the acquired GCPs was evaluated by using several measures with texture features of GCP chip images. This paper describes the details about proposed method for acquisition of GCPs and simulated results on relationship between texture features and GCP matching success rate corresponding to the cross correlation between reference and distorted GCP chip images.
\end{abstract}

Keywords-Ground Control Point: GCP; Digital Terrain Model: DTM; scattering model; complex dielectric constant; texture feature; matching success rate; GCP chip

\section{INTRODUCTION}

Various researches have been conducted on the method of extracting [1]-[5] Ground Control Point: GCP from image data obtained by optical sensors. In particular, these days, the technology in optical sensors can be effectively applied to image data by SAR (synthetic aperture radar) [6]. Have been shown in a study by Dr. Guindon et al [1]. They have proposed an automatic matching method between real and simulated SAR images with distortion due to terrain effects.

Essentially, space-based SAR imagery data has distortions due to layover, foreshortening, shadowing and so on. Therefore, geometric fidelity of the SAR imagery data is still key issues. One of the problems of geometric fidelity improvement of the space-based SAR imagery data is GCP extraction from the SAR imagery data. GCP extraction is not so easy. Also, another problem is matching accuracy improvement. By using GCPs, geometric distortions have to be removed through GCP matching. This is not so easy either. In order to improve these GCP extraction and GCP matching processes, GCP extraction from digital elevation model, and chip image based GCP matching are proposed here.

In this study, simulated a SAR image is generated using digital terrain model (DTM) and tried to extract GCP. In addition, as an evaluation of the extracted GCP, matching was performed between the GCP chip image with some distortion and the original image, and the accuracy was examined.
Furthermore, in order to evaluate the matching accuracy of the GCP chip using only the chip image, the correlation between the texture features and the accuracy was examined [7].

In this paper, the author first describes the simulation method for the SAR image adopted in this study, and then introduce the features introduced as indices for GCP evaluation [8]. Finally, the extracted GCPs are evaluated using these characteristic quantities, and the conditions for the optimal GCP are examined.

In the following section, related research works are described. Then, the proposed method is described followed by experimental set-up together with experimental results. After that, concluding remarks and some discussions are described.

\section{RELATED RESEARCH WORKS}

Automated matching of real and simulated SAR image as a tool for GCP acquisition is proposed [1]. Image-scale and look direction effect on the detectability of lineaments in radar images is studied and well reported [2]. Studies on GCP matching of remote sensing image data is conducted [3]. Accuracy of digital elevation data by various interpolation methods is assessed [4].

A method for textural analysis of synthetic aperture radar images by Grey Level Co-occurrence Matrix: GLCM method is proposed [5]. Also, Synthetic Aperture Radar: SAR is well overviewed [6]. Landsat Thematic Mapper: TM image classification using seasonal change of texture information is proposed [7]. Outline of national land numerical information is well organized [8].

Some studies on GCP selection process and its accuracy is investigated [9] together with effects on GCP success rate [10]. On the other hand, experiment on GCP matching is conducted and well reported [11] together with studies on GCP matching of remote sensing imagery data [12].

GCP acquisition using simulated SAR derived from Digital Elevation Model: DEM is attempted [13] together with GCP acquisition using simulated SAR and evaluation of GCP matching accuracy with texture features [14].

Effect of planimetric correction with a few GCPs on terrain height estimation with stereo pair is evaluated [15]. 
Meanwhile, speckle noise removal of SAR images with DEM is proposed [16]. Then, a method of speckle noise reduction for SAR data is proposed and tested [17]. After that, a new method for SAR speckle noise reduction (Chi Square Filter) is proposed and evaluated its effectiveness [18].

Decomposition of SAR polarization signatures by means of eigen-space representation is proposed [19]. Also, SAR image classification based on Maximum Likelihood: MLH decision rule with texture features taking into account a fitness to the probability density function is well reported [20]. Meanwhile, polarimetric SAR image classification with maximum curvature of the trajectory in eigen space domain on the polarization signature is proposed [21] together with polarimetric SAR image classification with high frequency component derived from wavelet multi resolution analysis: MRA [22].

Comparative study of polarimetric SAR classification methods including proposed method with maximum curvature of trajectory of backscattering cross section in ellipticity and orientation angle space is conducted [23]. Multi-Resolution Analysis: MRA and its application to polarimetric SAR classification is proposed [24]. Recently, Sentinel 1A SAR data analysis for disaster mitigation in Kyushu is reported. [25]. Comparison of geometric features and color features for face recognition is conducted and discussed [26].

\section{PROPOSED METHOD}

\section{A. Generation of SAR Image}

The proposed method for simulation of SAR image is based on DTM or DEM. There are some sources of the DTM. It allows slope calculation in accordance with its mesh size or spatial resolution. Without consideration of geometric distortions derived from foreshortening, shadowing, layover, speckle noise, SAR image can be simulated using just slope calculated with DTM, satellite altitude and off-nadir angle (look angle).

In the proposed model, the backscattering coefficient is calculated according to the following procedure.

1) Calculate the slope between each point from DTM.

2) Calculate the angle of incidence of the radar wave from the positional relationship between the satellite and the observation point, and calculate the distribution of the backscattering cross section 6 a assuming equation (1).

$\sigma^{0} \propto I_{r}=I_{n} \cos ^{2} \theta$

where $I r, I n$, and $\theta$ are the scattering direction unit vector, the scattering plane normal vector, and the radar wave incident angle, respectively.

3) Since the observation point is distorted in the distance between pixels due to the terrain effect, Lissasoprisog is performed at right angles using the following equation.

$$
\begin{aligned}
& P(i)=L_{i} \delta_{i}+L_{i-1}\left(1-\delta_{i-1}\right)+L_{i+1}\left(1-\delta_{i+1}\right) \\
& \delta_{i}=\left(x-\left|\frac{x}{2}-\delta x_{i}\right|\right) / x
\end{aligned}
$$

where $P(i)$ : i-th pixel value, $L i$ : i-th pixel value before resampling, $x$ : distance between pixels, $\delta x i$ : Pixel shift due to terrain effect.

Pixel shift $\delta x i$ due to the effect of layover, foreshortening, and shadowing was calculated from topographic information, and linear interpolation was performed by equation (2). However, the addition of a few terms in equation (2) was performed after judging whether or not $x$ / 2- $\Delta x i$ contributed to the i-th pixel value.

4) Convert the obtained values of $P i(X, Y) X, Y=1$ to 320 into 8-bit gray level image data of 0 to 255 .

\section{B. Extraction of $G C P$}

In order to extract GCP chip images from the simulated SAR image, the SAR image is divided with small size of subimages as candidates of GCP. Then, variance of the candidates of GCP chip image is calculated. It is assumed that GCP chip image has a relatively large variance. The image obtained in this way was divided into chips of $32 \times 32$ pixels, and the variance of the pixel value of each chip was calculated.

The actual SAR image has a geometric distortion caused by the deviation of the position and attitude of the satellite or their estimation errors such as skew and rotation. In order to simulate them, a skew or rotation is applied to the original image obtained by extracting the GCP chip as geometric distortion, and the area correlation between the resulting distortion image and the original GCP chip image is calculated. Here, the skew and the rotation were given as the center of rotation of the GCP chip image as shown in Fig. 1.

\section{GCP Matching}

Detailed steps for matching are as follows,

1) A point corresponding to the center point of each GCP chip in the original image is defined as GCP, and skew distortion and rotation distortion are given around this point.

2) A search window of $48 \times 48$ pixels is provided on the original image side centering on GCP.

3) Shift the GCP chip by one pixel in the search window, calculate the area correlation of the overlapping part, and obtain the peak of the correlation coefficient.

Here, the peak of the correlation coefficient was obtained by extracting nine correlation coefficients centered on the pixel of interest and interpolating independently in the $X$ and $Y$ directions by a quadratic equation.

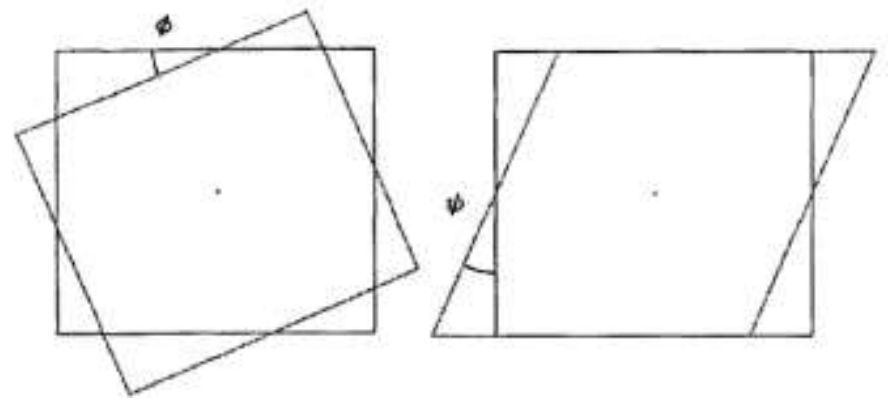

Fig. 1. Image Rotation $(\varphi)$ and Skew $(\Psi)$. 


\section{Texture Feature Extraction}

The author proposes a method for quantitatively evaluating the matching accuracy of GCP chips based on texture information. If the correlation between the matching accuracy and some feature amount of the chip image is strong, the GCP candidate with high automatic matching accuracy can be easily selected by evaluating the feature amount. In this study, feature extraction was performed using GLCM (Gray Level Co-occurrence Matrix) proposed by Haralick et. al (1973).

The 32 (PIXEL) x 32 (LINE) GCP chip consists of 256 gray levels, but for simplicity of calculation, this was integrated into 128 gray levels to calculate the GLCM. GLCM was obtained for four directions $\left(\theta=0^{\circ}, 45^{\circ}, 90^{\circ}, 135^{\circ}\right)$. GLCM was normalized by the following equations.

$P($ i.j.d, $\theta)=P(i, j . d, \theta) / R$

$R=\sum_{i=0}^{N_{q-1}} \sum_{j=0}^{N_{q-1}} P(i, j, d, \theta)$

where $P$ is the normalized co-occurrence probability matrix, and $P$ is the co-occurrence probability. The rate matrix (GLCM), d is the distance between pixels, and $N q$ is the number of gray levels.

Next, the following texture feature values were calculated from GLCM. Here, $P(i, j, d, \theta)$ is abbreviated as $P i j$.

i) Angular Second Moment

$\mathrm{ASM}=\Sigma \mathrm{i} \Sigma \mathrm{j} P j i^{2}$

ii) Homogeneity

$\mathrm{HOM}=\Sigma \mathrm{i} \Sigma \mathrm{j} P=i j /\{1+(\mathbf{i}-\mathbf{j})\}^{2}$

iii) Contrast

$\mathrm{CON}=\Sigma \mathrm{i} \Sigma \mathrm{j}(i-j)^{2} p i j$

iv) Dissimilarity

$\mathrm{DIS}=\Sigma \mathrm{i} \Sigma \mathrm{j}|i-j| P i j$

v) Entropy

$\mathrm{ENT}=-\Sigma \mathrm{i} \Sigma \mathrm{j} P i j \log P i j$

vi) Correlation coefficient (Correlation)

$\mathrm{COR}=\{\Sigma \mathrm{i} \Sigma \mathrm{j}(i j) P i j-\mu x \mu y\} / \sigma x \sigma y$

vii) Chi-square

$$
\mathrm{CHI}=\Sigma \mathrm{i} \Sigma \mathrm{j} P i j^{2} / P x(i) P y(j)
$$

where, $P i j$ is the average and standard deviation of the pixel values in the co-occurrence probability matrix direction, and $P x(i) P y(j)$ is the appearance probability in the matrix direction, respectively.

\section{EXPERIMENT}

\section{A. Generation of SAR Image}

The DTM used for the simulation is a magnetic tape file (KS110) produced by the Geospatial Information Authority of Japan. In this file, altitude data is stored in $\mathrm{m}$ units according to a format called a standard area mesh system. The primary area division mesh included in this data has a size of $80 \mathrm{~km}$ by $80 \mathrm{~km}$, which is equivalent to one terrain map of 20 planes, and is large enough as an East area. The section of code No.5338 where all the data of the grid points were prepared was selected from these. This is the area that includes Mt. Fuji in the lower right as shown in Fig. 2. Fig. 3 shows the simulated SAR image (Full scene) derived from the DTM.

The data is divided into $320 \times 320$ primary partitions, and arranged in a complicated manner according to the mesh code system, and is converted into a two-dimensional image. Since the data is divided into primary partitions of $320 \times 320$ and arranged in a complicated manner according to the mesh code system, it is difficult to process it as a two-dimensional image. In addition, in this data, since there are several abnormal grid points without elevation value as inland waters, they were replaced with the average value of two points before and after. Fig. 4 shows an example of a sub scene of the simulated SAR image.

Furthermore, the altitude values from 0 to $3776 \mathrm{~m}$ were requantized to 255 gray levels for processing as monochromatic images. The grid point interval of this mesh is $250 \mathrm{~m}$ on the actual ground, but it is coarse compared to the resolution, so the upper left corner was cut out as a subscene (Northern Akaishi Mountains), and interpolation was performed at $50 \mathrm{~m}$ intervals with a cubic spline function.

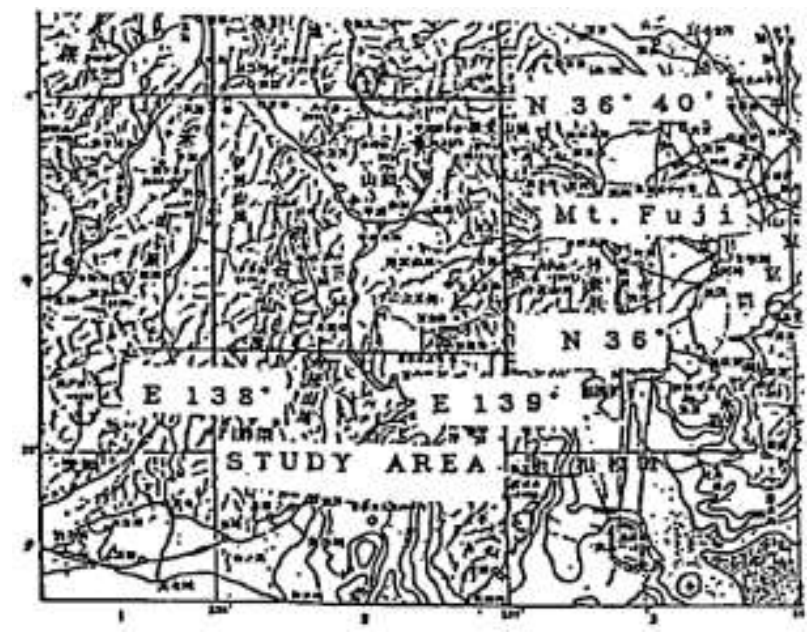

Fig. 2. Topographic Map of Intensive Study Area.

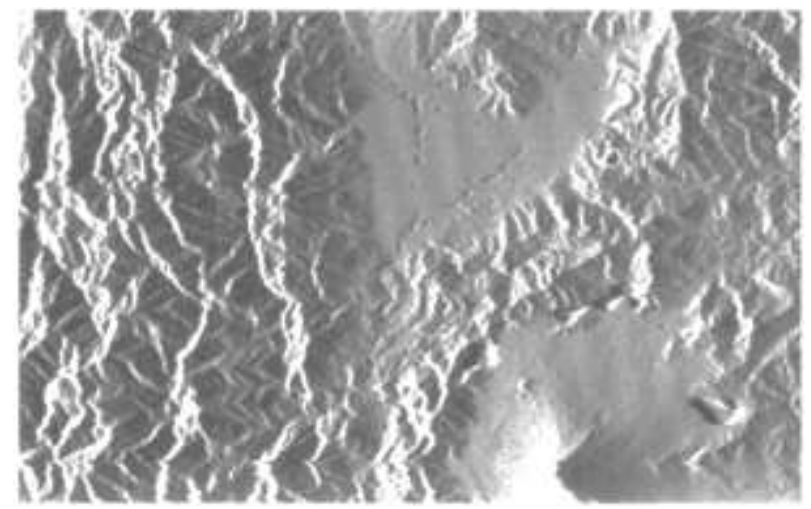

Fig. 3. Simulated SAR Image of Mt.Fuji and its Vicinity Derived from the DTM. 


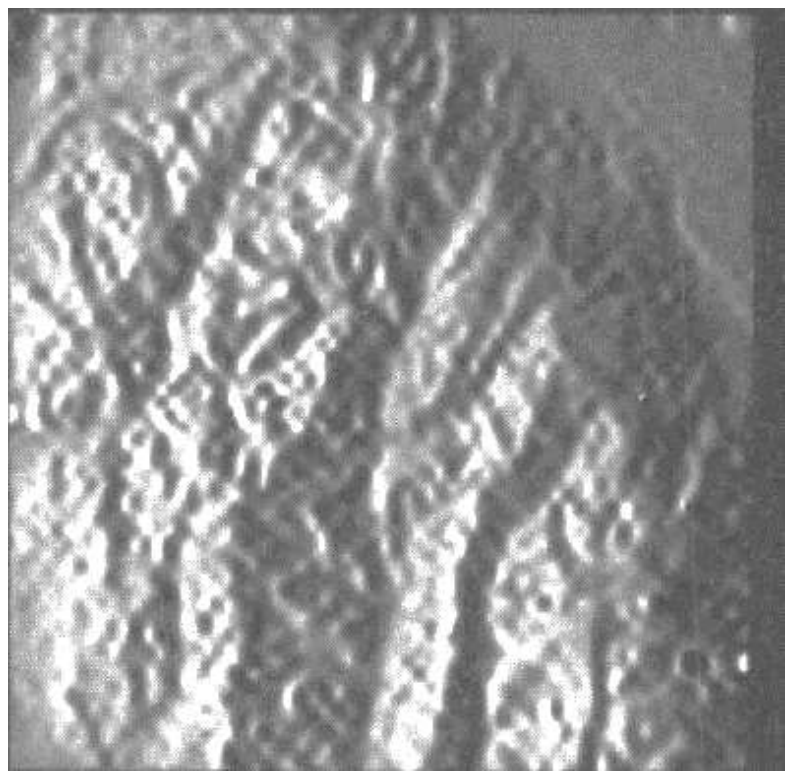

Fig. 4. Sub Scene of the Simulated SAR Image.

\section{B. GCP Extraction}

Since the observation area is a mountain area covered mostly by trees, it is considered to be a perfect diffusion surface, and it is assumed that the physical backscattering coefficient is uniform, and the reflected power obeys Rampart's cosine law. As the observation conditions from the satellite, as shown in Fig. 5.

It is assumed that observation is performed from west of the test site at an altitude of $570 \mathrm{~km}$ and an off-nadir angle of $35^{\circ}$ while flying north-south.

The images obtained in this way are divided into chips of $32 \times 32$ pixels, and the results of calculating the variance of the pixel values of each chip are shown in Fig. 6, 7. Of these, 14 chips shown in Fig. 6, 7 with a tether were randomly selected as GCP candidates. Figure 8 shows three-dimensional images of these chips.

\section{Texture Feature of GCP Chip Image}

Table I shows the matching accuracy of each chip when the skew and rotation were changed every $1^{\circ}$ from $1^{\circ}$ to $4^{\circ}$, respectively. The matching accuracy is the Euclidean distance between the estimated GCP indicated by the peak of the correlation coefficient and the true position. Skew and rotation can occur simultaneously in the real image, respectively, but each case is treated independently to separate and evaluate. Miss-identification is defined as the sum up of distances, between GCP chip center and functional correlation peak point, for which skew or rotation distortions from 1 to 4 degrees with $1 \mathrm{deg}$. step are added to the original image. Here, $R_{1}$ and $R_{2}$ correspond to, respectively, skew and rotation.

Table II shows the relationship between GCP matching accuracy and each feature value by correlation coefficient.

As an example, Fig. 9 shows the relationship between the magnitude of GCP mismatch due to skew distortion and GLCM CON. Here, the sizes of the mismatches from 1 to 4 were added and evaluated. Clearly a negative correlation is observed. Almost the same results were obtained for the other feature values. When the texture features in four directions were adjusted, it was found that there was a difference in the texture features in the $90^{\circ}$ direction compared to the other three directions, as shown in Table III.

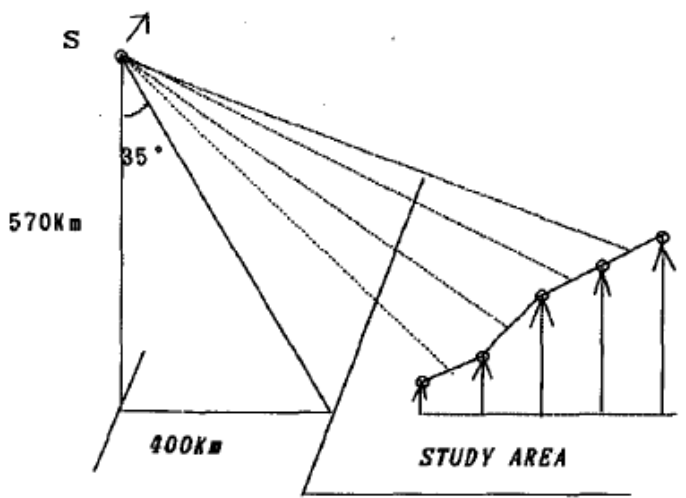

Fig. 5. Geometric Relationship between Satellite and Target.

\begin{tabular}{|c|c|c|c|c|c|c|c|c|c|}
\hline $1 x+8$ & $6 \cos _{3}$ & 25 & 382 & 25 & 145 & 188 & 273 & 260 & 211 \\
\hline 1224. & 8329 & 79 & 74 & 100 & 223 & 286 & 284 & 387 & 528 \\
\hline 198 & 623 & 343 & 31 & 142 & 215 & 225 & 308 & 275 & 346 \\
\hline 346 & 848 & 336 & 126 & $B 4$ & 246 & 139 & 280 & 254 & 225 \\
\hline 406 & 844 & 752 & 281 & 81 & 103 & 131 & 124 & 276 & 238 \\
\hline 709 & 1082 & 272 & 540 & 69 & 15 & 32 & 162 & 224 & 153 \\
\hline 675 & 954 & 415 & 634 & 61 & 104 & 236 & 260 & 121 & 133 \\
\hline 740 & 1032 & 529 & 585 & 127 & 269 & 263 & 167 & 177 & 204 \\
\hline 1092 & 474 & 873 & 408 & 212 & 671 & 50 & 8 & 58. & 134 \\
\hline 858 & 766 & 920 & 791 & 421 & 346 & 439 & 150 & 45 & 42 \\
\hline
\end{tabular}

This area is selected for subscene

Fig. 6. Gray Level Variance of each GCP Chip of Full Scene Divided into 10 by 10 Subsets.

\begin{tabular}{|c|c|c|c|c|c|c|c|c|c|}
\hline 165 & 32 & 25 & 80 & 181 & 165 & 64 & 3 & 5 & 85 \\
\hline 58 & 61 & 141 & 189 & 119 & 198 & 81 & $48 B$ & 13 & 235 \\
\hline 106 & 150 & 214 & $3 \times 2$ & $58 B$ & 130 & 163 & 28 & 42 & 251 \\
\hline 131 & 162 & 265 & 185 & 206 & 252 & 82 & 65 & 41 & 265 \\
\hline 124 & 227 & $2 \theta 2$ & 253 & 301 & 122 & 169 & 88 & 41 & 249 \\
\hline 183 & 178 & 340 & 305 & 139 & 85 & 178 & 118 & 137 & 406 \\
\hline 78 & 254 & 174 & 137 & 242 & 131 & 218 & 201 & 190 & 535 \\
\hline 58 & 208 & 234 & 83 & 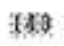 & $312:$ & 239 & 104 & 155 & 508 \\
\hline 58 & FE? & 285 & 365 & $8 x$ & 178 & \$us & 140 & 130 & 1037 \\
\hline 57 & 66 & 167 & 122 & 188 & 285 & 287 & 294 & 138 & 718 \\
\hline
\end{tabular}

W. This srea is selected for GCP's

Fig. 7. Gray Level Variance of each GCP Chip of Sub Scene Divided into 10 by 10 Subsets. 


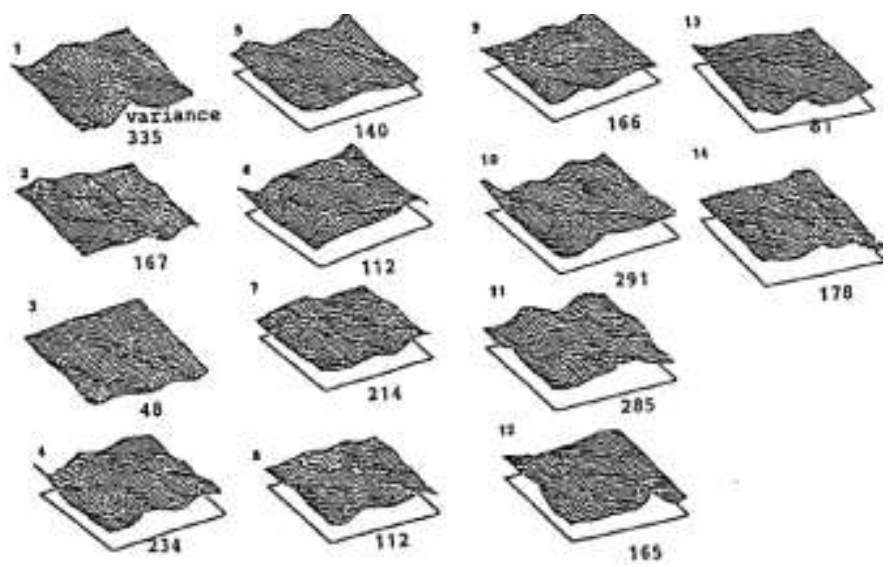

Fig. 8. Gray Level 30 Image and it's Variance of GCP Chip.

TABLE I. MISS-IDENTIFICATION DUE TO THE GEOMETRIC DISTORTIONS IN TERMS OF SKEW AND ROTATION

\begin{tabular}{|l|l|l|l|l|l|l|l|l|}
\cline { 2 - 9 } \multicolumn{1}{c|}{} & \multicolumn{4}{l}{ Skew Angle (deg.) } & \multicolumn{4}{l|}{ Rotation Angle (deg.) } \\
\hline GCP No. & 1 & 2 & 3 & 4 & 1 & 2 & 3 & 4 \\
\hline 1 & 0.065 & 0.156 & 0.256 & 0.364 & 0.073 & 0.168 & 0.259 & 0.363 \\
\hline 2 & 0.078 & 0.139 & 0.204 & 0.25 & 0.083 & 0.165 & 0.287 & 0.441 \\
\hline 3 & 0.106 & 0.212 & 0.34 & 0.468 & 0.149 & 0.313 & 0.481 & 0.67 \\
\hline 4 & 0.025 & 0.054 & 0.093 & 0.139 & 0.019 & 0.062 & 0.111 & 0.173 \\
\hline 5 & 0.07 & 0.125 & 0.195 & 0.272 & 0.026 & 0.08 & 0.138 & 0.2 \\
\hline 6 & 0.054 & 0.114 & 0.183 & 0.252 & 0.04 & 0.084 & 0.145 & 0.201 \\
\hline 7 & 0.024 & 0.067 & 0.127 & 0.19 & 0.085 & 0.156 & 0.228 & 0.286 \\
\hline 8 & 0.057 & 0.106 & 0.159 & 0.226 & 0.014 & 0.054 & 0.095 & 0.154 \\
\hline 9 & 0.025 & 0.051 & 0.088 & 0.128 & 0.102 & 0.212 & 0.33 & 0.464 \\
\hline 10 & 0.038 & 0.071 & 0.115 & 0.166 & 0.042 & 0.084 & 0.123 & 0.167 \\
\hline 11 & 0.026 & 0.041 & 0.07 & 0.095 & 0.028 & 0.058 & 0.092 & 0.127 \\
\hline 12 & 0.059 & 0.118 & 0.186 & 0.258 & 0.068 & 0.133 & 0.214 & 0.28 \\
\hline 13 & 0.077 & 0.115 & 0.226 & 0.306 & 0.048 & 0.087 & 0.131 & 0.179 \\
\hline 14 & 0.03 & 0.052 & 0.094 & 0.141 & 0.064 & 0.111 & 0.167 & 0.235 \\
\hline
\end{tabular}

TABLE II. CORRELATION BETWEEN EACH TEXTURE FEATURE AND MISIDENTIFICATION OF GCPS' DUE TO SKEW OR ROTATION

\begin{tabular}{|l|l|l|l|l|l|l|l|l|}
\hline & VAR & CON & CHI & ENT & ASM & HOM & DIS & COR \\
\hline R1 & -0.488 & -0.706 & -0.162 & 0.742 & 0.757 & 0.74 & -0.728 & 0.732 \\
\hline R2 & -0.29 & -0.61 & -0.323 & 0.562 & 0.626 & 0.627 & -0.628 & 0.53 \\
\hline
\end{tabular}

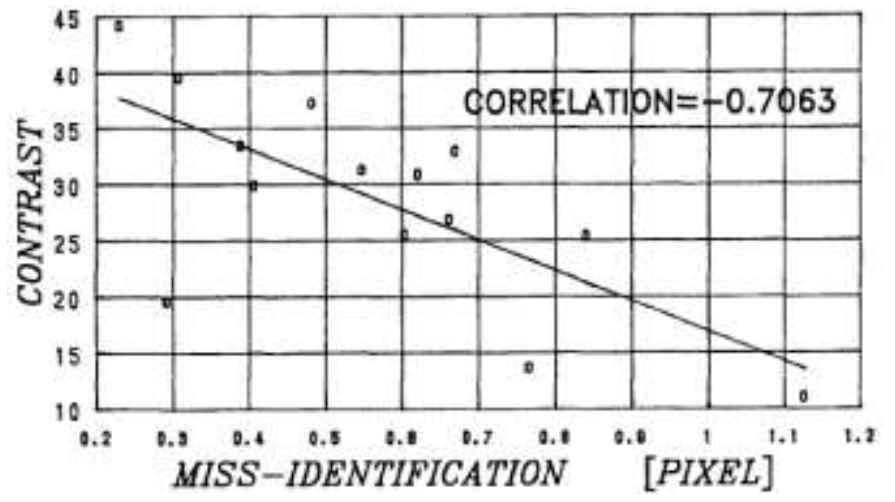

Fig. 9. Correlation between Contrast and Miss Identification (1-4 Degrees).
TABLE III. TEXTURE FEATURES OF GCP CHIPS

\begin{tabular}{|l|l|l|l|l|l|l|l|l|}
\hline GCP No. & $\sigma 2$ & CON & CHI & ENT & ASM & HOM & DIS & COR \\
\hline 1 & 335 & 25.33 & 30.43 & -21.91 & 0.0227 & 1.79 & 7.07 & 0.0459 \\
\hline 2 & 167 & 32.82 & 22.65 & -21.5 & 0.0298 & 1.64 & 8.25 & 0.0854 \\
\hline 3 & 48 & 10.98 & 14.66 & -17.5 & 0.0784 & 2.2 & 4.76 & 0.29 \\
\hline 4 & 234 & 39.5 & 20.82 & -22.3 & 0.0224 & 1.52 & 8.94 & 0.0829 \\
\hline 5 & 140 & 26.75 & 20.84 & -21.13 & 0.03 & 1.57 & 7.87 & 0.103 \\
\hline 6 & 112 & 25.5 & 22.11 & -20.44 & 0.0342 & 1.72 & 7.27 & 0.13 \\
\hline 7 & 214 & 28.78 & 19.81 & -21.27 & 0.293 & 1.55 & 8.25 & 0.0694 \\
\hline 8 & 112 & 31.15 & 25.71 & -20.54 & 0.0358 & 1.61 & 7.98 & 0.123 \\
\hline 9 & 166 & 19.56 & 20.83 & -20.82 & 0.0306 & 1.84 & 6.44 & 0.0891 \\
\hline 10 & 291 & 33.39 & 32.99 & -22.17 & 0.0208 & 1.16 & 8.23 & 0.0518 \\
\hline 11 & 285 & 44.13 & 18.92 & -23.09 & 0.0152 & 1.24 & 10.39 & 0.515 \\
\hline 12 & 165 & 30.81 & 27.09 & -21.42 & 0.0248 & 1.52 & 8.32 & 0.0889 \\
\hline 13 & 81 & 13.62 & 18.56 & -19 & 0.0502 & 2 & 5.47 & 0.184 \\
\hline 14 & 178 & 37.17 & 25.37 & -21.57 & 0.0291 & 1.54 & 8.76 & 0.0839 \\
\hline
\end{tabular}

\section{REMARKS}

From the experimental results, the following are remarks,

1) Table III shows that in this image, the 90 direction has less change than the other three directions. This is because the observation direction is 180 . It can be seen that these features are highly dependent on the beam irradiation direction in the SAR image.

2) Skew distortion has a higher correlation between each feature and matching success rate than rotation distortion. This is considered to be due to the fact that the rotation has more distortion (larger loss (change) of the original information) than the skew that deforms only in one direction even at the same angle.

3) Variance and chi-square values have low correlation with GCP matching accuracy. This is because they do not always correspond to the complexity of terrain change (BUSYNESS).

4) The contrast, dissimilarity, entropy, angular second moment and homogeneity show relatively strong negative or positive correlation with GCP mismatch degree. Therefore, a regression equation can be obtained from these correlations to predict the degree of GCP mismatch.

5) HOM, COM and DIS have cross-correlation, and ASM and ENT have high cross-correlation. Furthermore, there is a negative correlation between these groups. Therefore, it is desirable to evaluate GCP by selecting an appropriate one.

\section{CONCLUSION}

A method for extracting chip images suitable for GCP using simulated SAR images created from DTM was proposed. The followings are major results,

1) As a result of examining the relationship between the matching accuracy and the texture feature of the chip, the correlation with the index indicating the feature of GLCM of 
contrast, second moment of moment, entropy, homogeneity, dissimilarity is high It turns out. From this, it was found that by examining only the very small amount of texture of the chip image, GCP which seems to have high matching accuracy can be selected.

2) In the central Japan area (around Mt. Fuji) studied this time, the correlation between the matching accuracy and the texture feature amount is 0.7 to 0.75 when considering skew distortion, and 0.06 to 0.62 when similarly considering rotation distortion.

3) Among texture features, the angular second moment has the highest correlation with matching accuracy, which is suitable for GCP candidate selection.

\section{FUTURE RESEARCH WORKS}

The proposed system is adopted in the real earth observation satellite data, and it is a future subject to realize a more usable GCP acquisition method.

\section{ACKNOWLEDGMENT}

The author, also, would like to thank Professor Dr. Hiroshi Okumura and Professor Dr. Osamu Fukuda for their valuable discussions.

\section{REFERENCES}

[1] B. Guindon, H. Maruyama, Automated Matching of Real and Simulated SAR Image as a Tool for GCP Acquisition. Canadian Journal of Remote Sensing, Vol 12.2 pp. 149/159, 1985.

[2] Y. Yamaguchi, Image-Scale and Look Direction Effect on the Detectability of Lineaments in Rader Images. Remote Sensing of Environment. Vol 17 pp. 117/127, 1985.

[3] K. Tsuchiya, K. Arai, K. Tanaka, Studies on Ground Control Points Matching of Remote Sensing Image Data. Proceedings of the 14th Symposium on Space Technology and Science. pp. 1321/1328, 1984.

[4] Kuzumaki, Shuji, Yokoyama, Ryuzo, The 6th Lecture by the Japan Remote Sensing Society on the Accuracy of Digital Elevation Data by Various Interpolation Methods Proceedings of the Society, p. 55/58, 1986.

[5] Hashimoto, G., Matsuo, Y., A method for textural analysis of synthetic aperture radar images by GCLM method. .7,4 pp. 25/35, 1987.

[6] Nobuyoshi Raino, Synthetic Aperture Radar, Remote Sensing Society of Japan Vol1,1 pp. 49/107, 1981.

[7] Kohei Arai, K., TM Image Classification Using Seasonal Change of Texture Information, Journal of the Japan Society of Photogrammetry, Vol. 26, 4, 1987.

[8] Ministry of Construction, National Institute of Land and Geography, Department of Map Management, Outline of National Land Numerical Information, 1983.

[9] K.Tsuchiya, and Kohei Arai, Some studies on GCP selection process and its accuracy, Proc.of the 2nd Australasian Conference on Remote Sensing, 6-14-1-6.14.6, 1980.

[10] K.Tsuchiya and Kohei Arai, Effcts on GCP success rate, Proc.of the 7th Canadian Symposium on Remote Sensing, No.17, 497-501, 1980.

[11] K.Tsuchiya, Kohei Arai, and T.Tanaka, Experiment on GCP matching, Proc.of the ISPRS Commission II-3, and 4, B-18, 9-16 1983.

[12] K.Tsuchiya Kohei Arai and T.Tanaka, Studies on GCP matching of remote sensing imagery data, Proc.of the ISTS, m-3-2,1321-1328, 1984.

[13] Kohei Arai, and N.Fujimoto, GCP acquisition using simulated SAR derived from DEM, Proc.of the ISPRS Symposium, 33-40, 1988.

[14] Kohei Arai, GCP Acquisition Using Simulated SAR and Evaluation of GCP Matching Accuracy with Texture Features, International Journal of Remote Sensing, Vol.12, No.11, pp.2389-2397, Oct.1991.
[15] Kohei Arai, S.Mukai and Y.Terayama, Effect of planimetric correction with a few GCPs on terrain height estimation with stereo pair, Proc.of the ISPRS Committee-IV, PS-5-5, 1992.

[16] H.Wakabayashi and Kohei Arai, Speckle noise removal of SAR images with Digital Elevation Model: DEM, Proc. of the 5th ISCOPS Symposium, 1993.

[17] H. Wakabayashi and Kohei Arai, A method of Speckle Noise Reduction for SAR Data, International Journal of Remote Sensing, Vol.17, No.10, pp.1837-1849, May 1995.

[18] H. Wakabayashi and Kohei Arai, A New Method for SAR Speckle Noise Reduction(Chi Square Filter), Canadian Journal of Remote Sensing, Vol.22, No.2, pp.190-197, Jun.1995.

[19] Kohei Arai Decomposition of SAR Polarization Signatures by Means of Eigen-Space Representation, Proc. of the Synthetic Aparture Radar Workshop '98, 1998.

[20] Kohei Arai and Y.Terayama, SAR image classification based on Maximum Likelihood Decision rule with texture features taking into account a fitness to the probability density function, Final Report of JERS-1/ERS-1 System Verification Program, J2, vol.II, pp.2-415 to 424, Munich, Mar., 1995.

[21] Kohei Arai and J.Wang, Polarimetric SAR image classification with maximum curvature of the trajectory in eigen space domain on the polarization signature, Advances in Space Research, 39, 1, 149-154, 2007.

[22] Kohei Arai, Polarimetric SAR image classification with high frequency component derived from wavelet multi resolution analysis: MRA, International Journal of Advanced Computer Science and Applications, 2, 9, 37-42, 2011.

[23] Kohei Arai Comparative study of polarimetric SAR classification methods including proposed method with maximum curvature of trajectory of backscattering cross section in ellipticity and orientation angle space, International Journal of Research and Reviews on Computer Science, 2, 4, 1005-1009, 2011.

[24] Kohei Arai, Wavelet Multi-Resolution Analysus and Its Application to Polarizatic SAR Classification, Proceeding of the SAI Computing Conference 2016.

[25] Kohei Arai, Sentinel 1A SAR Data Analysis for Disaster Mitigation in Kyushu, Kyushu Brunch of the Japanese Society on Remote Sensing, Special Lecture for Young Engineers on Remote Sensing, Nagasaki University, 2018.

[26] Cahya Rahmad, Kohei Arai, Rosa A. Asmara, Ekojono Dimas, R. H. Putra, Comparison of Geometric Features and Color Features for Face Recognition, International Journal of Intelligent Engineering and Systems, IJIES on Volume 14, Issue 1, 541-551, 2021 DOI: $10.22266 /$ ijies.

\section{AUTHOR's PROFILE}

Kohei Arai, He received BS, MS and PhD degrees in 1972, 1974 and 1982, respectively. He was with The Institute for Industrial Science and Technology of the University of Tokyo from April 1974 to December 1978 also was with National Space Development Agency of Japan from January, 1979 to March, 1990. During from 1985 to 1987, he was with Canada Centre for Remote Sensing as a Post Doctoral Fellow of National Science and Engineering Research Council of Canada. He moved to Saga University as a Professor in Department of Information Science on April 1990. He was a councilor for the Aeronautics and Space related to the Technology Committee of the Ministry of Science and Technology during from 1998 to 2000. He was a councilor of Saga University for 2002 and 2003. He also was an executive councilor for the Remote Sensing Society of Japan for 2003 to 2005. He is a Science Council of Japan Special Member since 2012. He is an Adjunct Professor of University of Arizona, USA since 1998. He also is Vice Chairman of the Science Commission "A" of ICSU/COSPAR since 2008 then he is now award committee member of ICSU/COSPAR. He wrote 55 books and published 620 journal papers as well as 450 conference papers. He received 66 of awards including ICSU/COSPAR Vikram Sarabhai Medal in 2016, and Science award of Ministry of Mister of Education of Japan in 2015. $\mathrm{He}$ is now Editor-in-Chief of IJACSA and IJISA.http://teagis.ip.is.sagau.ac.jp/index.html. 